\title{
Issues in the modeling of carbon nanotube FETs: Structure, gate thickness, and azimuthal asymmetry
}

\author{
D. L. John · D. L. Pulfrey
}

Published online: 9 December 2006

(C) Springer Science + Business Media, LLC 2007

\begin{abstract}
Factors affecting the modeling of practical carbon nanotube field-effect transistors are addressed, namely: noncoaxial geometries such as the double-planar gate, and the semi-cylindrical gate; the thickness of the gate metalization; the azimuthal variation of the potential and the current. The $p$ $i$ - $n$ device is used to illustrate the importance of these factors.
\end{abstract}

Keywords Carbon nanotube FETs · Gate-thickness · Azimuthal variations in potential and current · Current operators

\section{Introduction}

The first phase of modeling carbon nanotube field-effect transistors (CNFETs) can be considered to be over: simplifying assumptions about the geometry (coaxial), transport (ballistic), the potential distribution (azimuthally invariant), and the gate-metal thickness (zero), have allowed "ultimateperformance" predictions to be made for both DC and AC operation. Researchers are now examining these assumptions in order to determine whether they are appropriate for models intended to predict, and analyze, the performance of more realistic devices. In this work we focus on the azimuthal asymmetry of both the potential and the local current; and on the gate-metal thickness in two, non-coaxial structures. For illustrative purposes, we examine the $p-i-n$ doped-contact CNFET. This device is of particular interest

D. L. John $(\bowtie) \cdot$ D. L. Pulfrey

Department of Electrical and Computer Engineering, University

of British Columbia, Vancouver, BC V6T1Z4, Canada

e-mail: davej@ece.ubc.ca

D. L. Pulfrey

e-mail: pulfrey@ece.ubc.ca because it appears capable of an exceptional sub-threshold slope [1]. We self-consistently solve the equations of Poisson and Schrödinger: the latter gives the local density of states from a $p_{z}$-tight-binding Hamiltonian using a Green's Function $^{1}$ approach, within which the self-energies for the semi-infinite leads are computed by solving a quadratic matrix equation [3]. We consider two structures: the semicylindrical-gate geometry (SC) shown in Fig. 1(a), which may be a realizable tri-gate-like structure, and an asymmetrical, double-gate geometry (DG) shown in Fig. 1(b), which has recently been used to study doped-contact CNFETs [4].

\section{Current operator}

In reality, the perfection of the carbon nanotube molecule may be disrupted by defects or foreign atoms. Such imperfections may affect the current in a CNFET and might lead to interesting sensor applications. Here, we take a step towards being able to analyze, on the inter-atomic level, such a situation by deriving the real-space current operator for a perfect nanotube. Figure 2 shows the hexagonal lattice, and identifies the two atoms ( $h$ and $l$ ) in the primitive cell. Let $|\Psi\rangle$ be a column vector of the wavefunctions for each of the atoms in our system, and $\langle\Psi|$ be a row vector of the conjugates of each of the wavefunctions. The Schrödinger Wave Equation and its conjugate can then be combined to yield

$$
\begin{aligned}
\frac{\partial}{\partial t}(|\Psi\rangle\langle\Psi|) & =\frac{1}{i \hbar}\left(H|\Psi\rangle\langle\Psi|-| \Psi\rangle\langle\Psi| H^{\dagger}\right), \\
& \equiv-\nabla \cdot \tilde{\mathbf{J}},
\end{aligned}
$$

\footnotetext{
${ }^{1}$ Note that the term "Non-Equilibrium Green's Function" has also been used to describe this formalism even in situations where self-energies due to scattering are not included, see Ref. [2] for example.
} 
Fig. 1 The (a) semi-cylindrical and (b) double-gate structures, showing the gate, and the nanotube, with the lateral, doped regions in the lighter shade

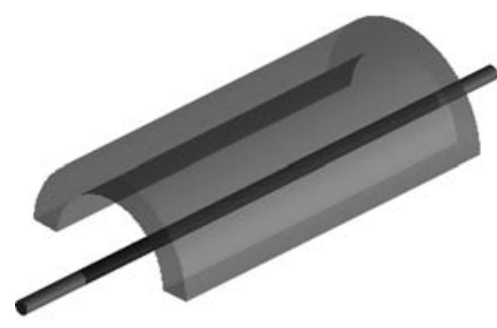

(a)

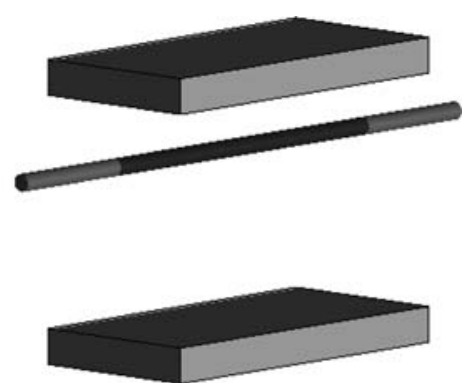

(b) where the second equation defines a probability density current, with the tilde indicating that $\tilde{\mathbf{J}}$ is evaluated for a particular energy, and that an integration over $E$ will eventually be required to get the total current density $\mathbf{J}$. Considering atom $h$, then the divergence of $\tilde{\mathbf{J}}$ over its bounding equilateral triangle is

$\nabla \cdot \tilde{\mathbf{J}}^{h} \simeq \frac{4}{3 a}\left(\tilde{J}_{\rightarrow}^{h}+\tilde{J}_{\nwarrow}^{h}+\tilde{J}_{\swarrow}^{h}\right)$,

where $a$ is the inter-atom spacing, the subscript indicates the direction of the surface through which the current passes, and $\tilde{J}$ is the magnitude of the specified component of the current density. Using a tight-binding Hamiltonian, and considering only nearest-neighbour interactions, we obtain

$$
\begin{aligned}
\frac{\partial}{\partial t}\left[\Psi^{h}\left(\Psi^{h}\right)^{\dagger}\right]= & \frac{\gamma}{i \hbar}\left[\Psi_{\rightarrow}^{h}\left(\Psi^{h}\right)^{\dagger}-\Psi^{h}\left(\Psi_{\rightarrow}^{h}\right)^{\dagger}+\Psi_{\nwarrow}^{h}\left(\Psi^{h}\right)^{\dagger}\right. \\
& \left.-\Psi^{h}\left(\Psi_{\nwarrow}^{h}\right)^{\dagger}+\Psi_{\swarrow}^{h}\left(\Psi^{h}\right)^{\dagger}-\Psi^{h}\left(\Psi_{\swarrow}^{h}\right)^{\dagger}\right],
\end{aligned}
$$

where $\gamma$ is the overlap energy. Therefore we define

$\tilde{J}_{\rightarrow}^{h}=-\frac{3 \gamma a}{4 i \hbar}\left[\Psi_{\rightarrow}^{h}\left(\Psi^{h}\right)^{\dagger}-\Psi^{h}\left(\Psi_{\rightarrow}^{h}\right)^{\dagger}\right]$

and similarly for the other current densities.

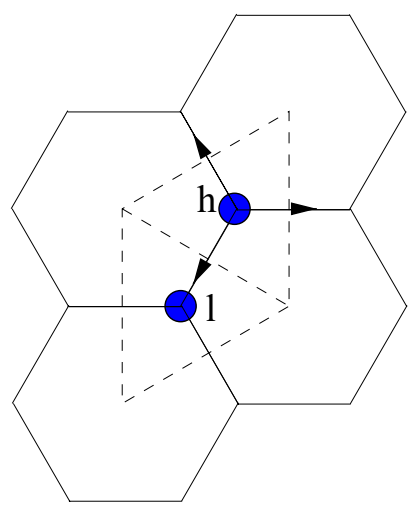

Fig. 2 Hexagonal lattice (solid) with a primitive cell (dashed parallelogram) divided into two equilateral triangles

\section{Results and discussion}

Results are presented for $p-i-n$ CNFETs fashioned from (16, 0 ) tubes with a doping density in the contact regions of $10^{-1} \mathrm{~nm}^{-2}$. The oxide is taken to be silica, with a thickness of $2 \mathrm{~nm}$, except for the lower insulator in the double-gate structure, where the thickness is $20 \mathrm{~nm}$. The gate metal thickness $T_{G}$ is a variable. Field-fringing from the edges of the gate affects the length $L_{S D}$ of the doped regions that must be employed in order to ensure charge neutrality at the ends of the contacts, where null-Neumann boundary conditions are taken to apply [2]. Illustrative results are shown for the semi-cylindrical device in Fig. 3, with charge neutrality being indicated by the potential becoming flat well before the end of the contact. For $L_{S D}=15 \mathrm{~nm}$, which was the value used here in the interests of computation time, it can be seen that the maximum $T_{G}$ we can use is $2 \mathrm{~nm}$. The double-gate case is less restrictive, and charge neutrality is obtained for $T_{G}$ up to $\approx 10 \mathrm{~nm}$ with our chosen $L_{S D}$. This effect of gate thickness should be borne in mind when practical devices with thicker gate metalization are being considered, e.g., in devices for high-speed switching, or high-frequency operation, where proper accounting of the inter-electrode capacitance is mandatory $[5,6]$.

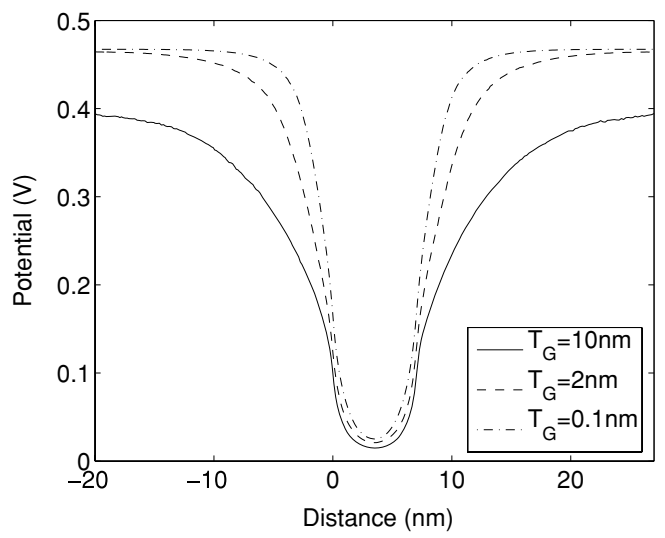

Fig. 3 Longitudinal variation of surface potential under equilibrium conditions: effect of gate thickness for the semi-cylindrical-gate CNFET 


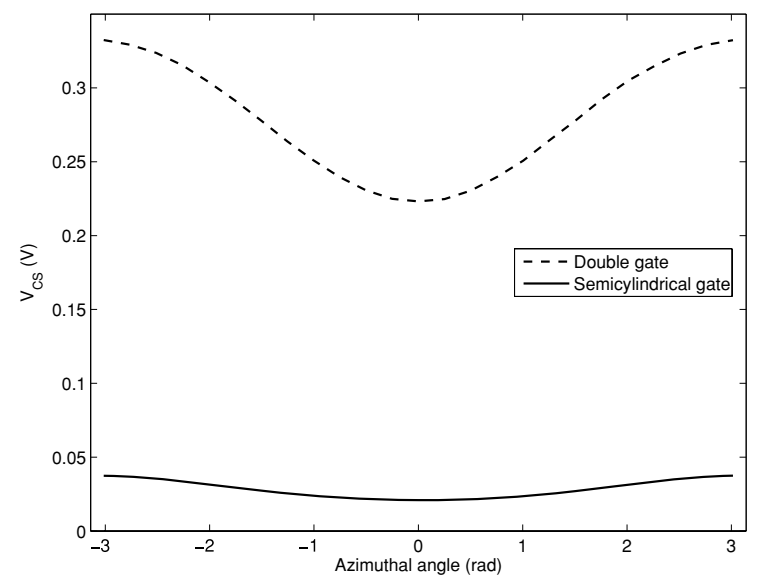

Fig. 4 Azimuthal variation of surface potential at mid-length of the nanotube for both structures under equilibrium conditions

Whereas Fig. 3 shows the longitudinal variation of potential at a fixed azimuth (the top of the tube), Fig. 4 shows the azimuthal variation in potential for our two structures at a fixed longitudinal position (the mid-length of the intrinsic part of the tube). As expected, the planar structure shows more azimuthal asymmetry.

To investigate the effect of this asymmetry under nonequilibrium conditions, consider the data for the double-gate device (open symbols) in the gate characteristic of Fig. 5. The data marked by triangles is for $T_{G}=5 \mathrm{~nm}$, but with the potential computed only on the axis of the tube, i.e., without consideration of any azimuthal variations. It is clear from this figure that the azimuthal variation in potential does not impact significantly on the terminal characteristics, at least for the structures considered here.

However, a significant fact revealed by Fig. 5 is that the thickness of the gate metal does markedly affect the drain

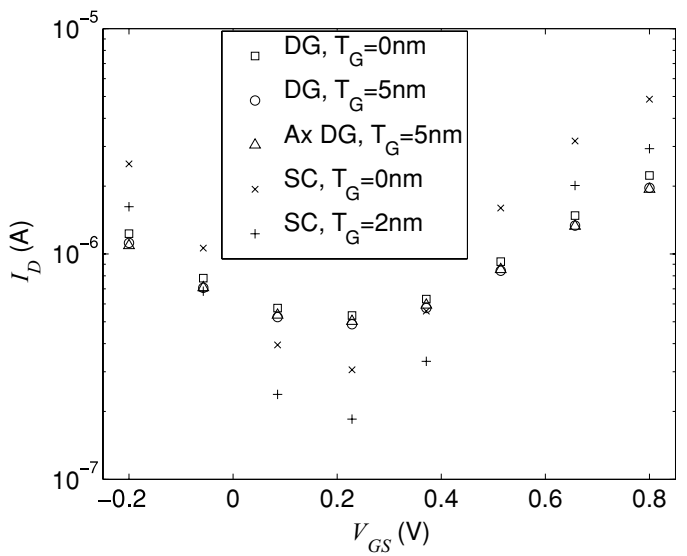

Fig. 5 Gate $I$ - $V$ characteristics at $V_{D S}=0.4 \mathrm{~V}$ for the two device structures, with various values of gate thickness $T_{G}$. All data sets consider the azimuthal variation in potential, except that of the open triangles, for which the potential on the tube was taken to be constant at its axial value

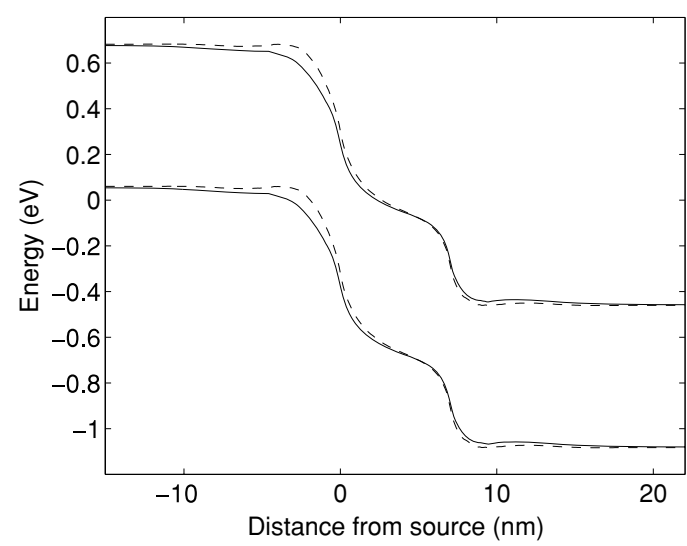

Fig. 6 Energy band diagram for the semi-cylindrical-gate CNFET at $V_{G S}=0.37 \mathrm{~V}$, and $V_{D S}=0.4 \mathrm{~V}$, showing the effect of metal-gate thickness for $T_{G}=0$ (dashed), and $T_{G}=2 \mathrm{~nm}$ (solid)

current in the case of the semi-cylindrical structure. This is because the fringing-field from the gate to the contacts affects the band-bending at the junctions of the contacts with the intrinsic portion of the tube. This is confirmed by the band diagram of Fig. 6: for the thinner gate, there is steeper bandbending at the source-end of the channel. This facilitates the inter-band tunneling of electrons, thereby increasing $I_{D}$.

The results of a current-operator computation are shown in Fig. 7. There is considerable structure in this plot, both in the azimuthal and longitudinal directions even though, as required, the sum of the currents around the circumference is independent of the longitudinal position. If we consider the case where the axial potential is used, rather than the proper surface potential, when simulating this device, the current will be distributed evenly around the $\mathrm{CN}$ circumference. In this case, there is about $\pm 20 \%$ variation in the inter-atomic current, despite the fact that the terminal current has only a weak dependence on azimuthal potential variations. Further work, involving imperfect nanotubes, and connections between dissimilar tubes, is planned, where the impact on the carrier flow may be investigated using this method.

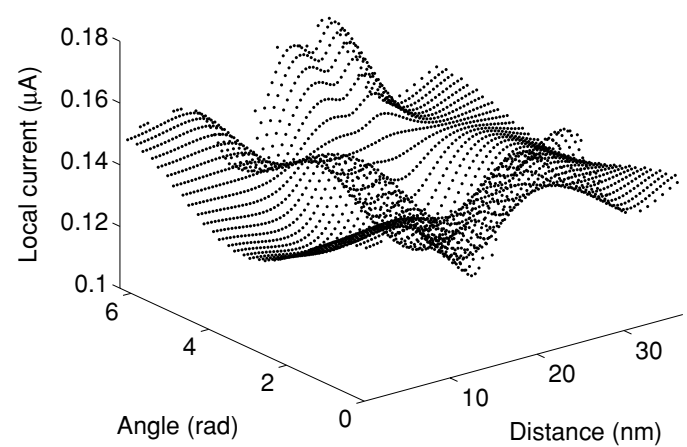

Fig. 7 Spatial variation of the current, as computed from the current operator integrated over energy, for the double-gate structure at $V_{G S}=$ $0.8 \mathrm{~V}$, and $V_{D S}=0.4 \mathrm{~V}$ 


\section{Conclusions}

From this simulation study of doped-contact CNFETs, it can be conluded that:

- To ensure charge neutrality at the ends of the doped contacts, the gate-metal thickness should be taken into account when choosing the lengths of the source and drain regions.

- In semi-cylindrical structures, the fringing fields from the gate can significantly affect the band-bending, particularly at the source/intrinsic-nanotube interface, thereby affecting inter-band tunneling and, consequently, the drain current.

- There is appreciable azimuthal variation in the surface potential of the nanotube in double-gate structures. However, this does not seem to affect significantly the terminal characteristics.

- The current-operator method reveals significant spatial structure in the current.
Acknowledgment The financial support of the Natural Sciences and Engineering Research Council of Canada is gratefully acknowledged.

\section{References}

1. Appenzeller, J. et al.: Comparing carbon nanotube transistors-the ideal choice: a novel tunneling device design. IEEE Trans. Elec. Dev. 52, 2568 (2005)

2. Datta, S.: Nanoscale device modeling: the green's function method. Superlattices and Microstructures 28, 253 (2000)

3. John, D.L., Pulfrey, D.L.: Green's function calculations for semi-infinite carbon nanotubes. Phys. Stat. Solidi B 243, 442 (2006)

4. Fiori, G., et al.: Performance of carbon nanotube field-effect transistors with doped source and drain extensions and arbitrary geometry. Tech. Digest IEDM, 529 (2005)

5. John, D.L., Pulfrey, D.L.: Switching-speed calculations for schottkybarrier carbon nanotube FETs. J. Vac. Sci. Technol. A 24, 708 (2006)

6. Castro, L.C., Pulfrey, D.L.: Extrapolated $f_{\max }$ for carbon nanotube FETs. Nanotechnology 17, 300 (2006) 